\title{
EL CINE COMO ESTRATEGIA DIDÁCTICA INNOVADORA Metodología de estudio de casos y perfil de estrategias docentes
}

\author{
Saturnino de la Torre, \\ Carme Oliver, \\ Verónica Violant, \\ José Tejada, \\ Nuria Rajadell \\ y Mercè Girona \\ Miembros del grupo EDIFID' \\ Formarse es reflexionar sobre la propia práctica para mejorarla.
}

(S. de la Torre)

RESUMEN: El presente estudio forma parte de una investigación más amplia basada en la integración de tres conceptos habitualmente estudiados por separado (Investigación, Formación, Innovación) que busca la mejora de la calidad de la enseñanza a partir del estudio de las estrategias didácticas. Los modelos basados en la mera reflexión no se han mostrado eficaces para el cambio docente así como tampoco los que se han quedado en la mera práctica, descontextualizada del lugar de trabajo. Por ello, esta investigación intenta dar sentido conjunto a estos tres conceptos: generación de conocimiento, formación e innovación. Se define el concepto de estrategia didáctica, (Torre, 1997, 2000) en base a sus seis componentes: perspectiva teórica, finalidad o meta perseguida, carácter adaptativo, realidad contextual, personas implicadas, y aspectos organizativos, funcionalidad y eficacia.

Se describe así mismo el modelo holodinámico de análisis en el contexto del aula (Torre, 2003) tomando en consideración ocho parámetros: supuestos implícitos del docente, contexto, rol docente, rol discente, recursos, organiza-

1. El grupo EDIFID (Estrategias didáctica innovadoras para la formación e innovación docente), que disfruta de la ayuda I+D del MCYT, está formado actualmente por profesores de la Universidad de Barcelona y Universidad Autónoma de Barcelona, Saturnino de la Torre (Coordinador), José Tejada, Inmaculada Bordas, María de Borja, M. Antonia Pujol, Carmen Oliver, Nuria Rajadell, Verónica Violant, Nacho Jarne, Mercè Girona (Becaria). Todos ellos han tenido un papel relevante en la realización de este trabajo. 
ción espacio-temporal, clima o interacción, evaluación. Estos son los indicadores que se toman en cuenta en el estudio de casos que se describe utilizando como soporte informativo el cine comercial y como técnica de representación los perfiles. El cine formativo es una estrategia que el grupo GAD de la Universidad de Barcelona viene utilizando desde hace diez años tanto en forums anuales como en las aulas. Por otra parte, la técnica de representar en perfiles una compleja información cualitativa facilita la comprensión global de aquellos puntos fuertes y débiles en la mejora de la docencia.

ABSTRACT: The present study is comprised in an ampler cradle investigation based on the integration of three concepts, usually studied separatedly (Research, Training, Innovation), in order to improve the quality of education thanks to the study of didactic strategies. Models' based reflection has not been effective for a teaching change as well as those that have been kept in the mere practice, out of the context of the work place. Thus, this investigation tries to give joint sense to these three concepts: knowledge generation, training and innovation. Didactic strategy concept (Torre, 1997, 2000) is defined based on its six components: theoretical perspective, purpose or pursued aim, adaptative character, contextual reality, implied people and organizational aspects, functionality and effectiveness.

Holodynamic analysis model is described in the classroom context (Torre, 2003) considering eight parameters: teacher's implicit assumptions, context, teacher rol, pupil rol, resources, time and space organization, interaction and evaluation. These are the indicators taken into account in the described study of cases using commercial cinema as informative support and the profiles as representation technique. The formative cinema is a strategy that has been used for ten years as much in annual forums as in classrooms by GAD group of the University of Barcelona. On the other hand, the technique of representing by profiles complex qualitative information facilitates the global understanding of the strong and weak points of the teaching improvement.

PALABRAS CLAVE: Estrategia didáctica, Cine formativo, Innovación, Estudio de caso, Perfiles de estrategias, Metodología de desarrollo.

KEYWORDS: Didactic strategy, Formative cinema, Innovation, Study of cases, Profiles strategy, Development Methodology.

\section{Introducción}

Nadie duda hoy que la enseñanza universitaria está en un momento de transformación y búsqueda de un nuevo sentido del conocimiento urgido por la realidad social y la demanda de calidad. La universidad humboliana del siglo XVIII está dando paso a un espacio más abierto y flexible que prepare personal y profesionalmente para la vida, que responda a los problemas que tiene la sociedad actual. Los procesos de cambio que afectan a la sociedad en general y a la educación en particular, la marcha imparable de la globalización económica y socialización del conocimiento, la progresiva introducción de los paradigmas ecosistémicos en las Ciencias Sociales (impulsados por autores como Maturana, Varela, Prigogine, Morin...), la eminente y acelerada presencia de la convergencia europea sobre la enseñanza universitaria nos hacen pensar que la creatividad debe tener un lugar destacado en este 
proceso de transformación. Si la creatividad se alimenta de problemas, crisis y situaciones de cambio, estamos en un momento propicio para recurrir a este potencial humano.

Las políticas universitarias toman en cuenta cada vez más los conceptos de calidad, de innovación, de enseñanza basada en el aprendizaje, pero sobre todo de que el profesorado ha de incorporar formas nuevas orientadas al desarrollo de habilidades y competencias del alumnado. El dominio de los contenidos pasa así a formar parte de lo que denominamos competencias, tanto personales como sociales y profesionales. Ello significa que la docencia está en proceso de transformación y que la transmisión de contenidos es una estrategia más que una finalidad. El peso de enseñar está en conseguir que el alumnado aprenda y, por tanto, las estrategias no son de transmisión sino de interacción, motivación, implicación, aplicación, investigación, tutoría, resolución de problemas, simulación... estrategias dentro y fuera del aula.

La imagen ideal del docente universitario no está tanto en el dominio del contenido, que ha de presumirse como paso previo a su actuación en el aula, así como otros supuestos implícitos que a continuación indicaremos, sino en el modo como planifica, organiza y logra que el alumno aprenda. No es únicamente el transmisor del conocimiento científico y profesional, sino el inductor de aprendizajes, habilidades y competencias, y por tanto, creador de condiciones apropiadas a los objetivos. Bajo estos supuestos resulta evidente que el dominio de estrategias didácticas variadas y la posesión de recursos tanto materiales como formales son determinantes del éxito y de la calidad docente. Es por ello que nuestra investigación pivota sobre el concepto de estrategia didáctica, ya que a través de la misma, se consigue innovar en la enseñanza de forma grata y motivante.

Si un profesional es una persona competente en su ámbito, capaz de analizar y resolver los problemas y proponer mejoras (innovar), el profesor/a universitario es un profesional de la enseñanza superior innovador y creativo, con dominio del contenido formativo y de estrategias didácticas, capaz de hacer que los alumnos se entusiasmen por aprender y logren las competencias deseadas. Esta sería la clave para plantear la acción docente en la universidad y por consiguiente un modelo de formación y análisis de cuanto sucede en el aula, como un espacio más de aprendizaje, pero no el único.

La creatividad, es el alma de las estrategias innovadoras orientadas al aprendizaje, por cuanto es el alumno el que ha de ir mostrando la adquisición de las competencias convenidas en cada una de las carreras. El autoaprendizaje es una consecuencia inmediata de esta nueva orientación y la evaluación formativa se transforma en evaluación formadora. Esto es una evaluación que garantiza su utilidad porque surge desde la reflexión del propio discente en lugar de hacerlo desde el docente.

Sin embargo, somos conscientes de que entre este ideal y la realidad existe una distancia, considerable. La metodología docente, en general sigue anclada en formas transmisivas. Es por ello que pretendemos indagar las modalidades estratégicas, y construir perfiles docentes teniendo en cuenta el tipo de materia, obligatoria u optativa, el turno, el tamaño del grupo, entre otros aspectos. Posiblemente nos encontremos con una heterogeneidad metódica, motivada más por las diferencias del profesorado que por los propios contenidos.

El aula es un escenario formativo, en el que convergen múltiples actores e indicadores, caracterizado por la complejidad, la interactividad, la enacción, el predo- 
minio de la intencionalidad sobre la espontaneidad, la comunicación, la pertenencia... Es el espacio en el que se construyen los conocimientos con mayor facilidad, por cuanto se dan las condiciones adecuadas para transformar la información en formación. Por eso, analizar lo que sucede en el aula a lo largo de una clase o de una asignatura, nos ayuda a entender no sólo las estrategias didácticas, sino los mecanismos de la calidad docente, de la innovación y, en cierto modo, de la formación pedagógica.

Antes de hacer una somera descripción de las principales dimensiones y componentes del modelo utilizado en el análisis del aula, no está de más plantearnos qué comporta ser profesional de la enseñanza y establecer el marco referencial de lo que entendemos por estrategia didáctica.

\section{2. ¿Qué comporta ser profesional de la enseñanza?}

Entender la enseñanza como una profesión y al profesor como profesional lleva implícito plantarse el papel que juega el conocimiento en la actividad docente. Un profesional tiene competencias no sólo para resolver problemáticas o situaciones concretas, sino que conoce el porqué y para qué de aquello en lo que se ocupa. No es un mero técnico, sino una persona reflexiva, capaz de analizar y mejorar su práctica. Posee una visión capaz de ir más allá del problema o situación, conecta la teoría, la técnica y la práctica. Es por ello que el docente, maestro o profesor, en tanto que profesional de la enseñanza ha de poseer unas competencias respecto al contenido, a la didáctica o forma implicar al alumno en su dominio y ser capaz de actualizarse y desarrollarse profesionalmente. Podría hablarse mucho sobre las connotaciones del docente como profesional, pero nos referiremos únicamente a tres aspectos.

a) En primer lugar, estar en posesión del conocimiento con un nivel satisfactorio. Es lo que pediríamos a cualquier profesional al que compramos su servicio. Que conozca aquello que nos vende, que posea el dominio o conocimiento suficiente sobre la materia. Un docente ha de estar no sólo informado, sino formado en el contenido que imparte y conocer la epistemología de dicho contenido, pues es muy distinta la enseñanza de lengua, sociales, matemática o psicología. Cada disciplina posee su estructura, lenguaje, método, terminología, y sobre todo una forma de construirse e investigarse.

b) En segundo lugar, actuar de forma didáctica, esto es tomar decisiones curriculares adaptadas a las características diferenciales de los sujetos. Esta afirmación tan simple tal vez sea una de las más complicadas de realizar en la práctica. Porque no se trata sólo de conocer el contenido, sino de seleccionarlo, secuenciarlo y proponer las actividades pertinentes con la madurez de los sujetos. Ello comporta tener conocimientos pedagógicos, didácticos y psicológicos. Es la formación psicopedagógica y didáctica que convierten en docente a un licenciado o persona que posee conocimientos sobre una determinada materia. Siendo más concretos, estar capacitado para resolver la problemática inherente a su profesión. Ello comportará saber tomar decisiones apropiadas, tanto por lo que se refiere a la planificación como al desarrollo curricular y la evaluación. Normalmente conocemos a un buen profesional, ya sea mecánico, médico o administrador, porque acierta fácilmente con el diagnóstico y con el tratamiento adecuado. Un docente innovador y creativo es 
capaz de estimular e implicar al alumnado en aquellos aprendizajes relevantes de la materia.

c) En tercer lugar, poseer la formación y disposición para mejorar profesionalmente mediante la autoformación, la reflexión crítica sobre su práctica y la realización de proyectos de innovación. Este rasgo es el que se relaciona más directamente con la idea del profesor como profesional innovador y creativo por cuanto ha de ir más allá de lo aprendido para incorporar nuevas ideas en su forma de enseñar y actuar. Es capaz de reflexionar sobre su práctica para mejorarla. El desarrollo profesional del docente comienza a trasladarse al ámbito universitario, como lo ponen de manifiesto los trabajos de P. de Vicente (2002), C. Marcelo (1995), L.M. Villar (1999), J. Tejada (2000, 2002)

\section{Un marco teórico para definir la estrategia didáctica}

Si el método es un término deudor de la reflexión filosófica, por cuanto es una vía ascendente o descendente entre la teoría y la práctica. Si la técnica es deudora del enfoque positivo y responde a la secuencia encadenada de acciones que facilitan la consecución eficaz del objetivo; si el procedimiento es una forma abierta y aproximativa para acercar metas y logros; el término estrategia lo utilizamos con preferencia por responder mejor a un enfoque interactivo y ecosistémico. La realidad social, educativa, creativa no son lineales, ni rígidas, ni estáticas, sino, por el contrario, se caracterizan por ser complejas, adaptativas, cambiantes, interactivas, deudoras de entornos y contextos socioculturales. Es por ello que el concepto de estrategia responde mejor a nuestros propósitos, entendida como procedimiento adaptativo o conjunto de ellos por el que organizamos secuenciadamente la acción para lograr el propósito o meta deseado.

Un concepto amplio, abierto, flexible, interactivo y sobre todo adaptativo, aplicable tanto a la concreción de modelos de formación, de investigación, de innovación educativa, de evaluación, docencia o estimulación de la creatividad. Las estrategias nos acompañan siempre haciendo de puente entre metas o intenciones y acciones para conseguirlos.

Dicho concepto comporta, a nuestro entender, los siguientes componentes: (S. De la Torre, 2000, 112ss)

a) Una consideración teórica o perspectiva de conjunto del proceso. La estrategia añade a otros conceptos el hecho de poseer una legitimación y enfoque que proporciona direccionalidad y visión de conjunto a las acciones concretas a realizar. La estrategia implica un por qué y para qué. No se trata de una réplica automática al estilo de la técnica, ni la búsqueda de la eficacia en sí, sino de su pertinencia con los valores dominantes y la ética que justifica o no determinadas actuaciones. En ocasiones ha de renunciarse a la eficacia de acuerdo con criterios éticos o de valor. Así una crítica, en un momento determinado, en caliente, puede parecer eficaz para producir el cambio, pero no ser recomendable por razón de impacto negativo, de inhibición o bloqueo que pueda tener sobre el sujeto. De hecho, este tipo de conductas han sido frecuentes en la educación.

b) Un segundo componente es la finalidad o meta deseada. La estrategia, al igual que el método o procedimiento, y cualquier actuación formativa, encuentran 
su razón de ser en la meta perseguida. Incluso los grupos y organizaciones se cohesionan y mantienen en el tiempo gracias a compartir determinados fines que intentan conseguir. Alcanzados estos, el grupo pasa por momentos de crisis y descomposición. Las estrategias se concretan a la luz de las finalidades y objetivos. Por eso carece de sentido hablar de la bondad o pertinencia de las estrategias en general, al margen de lo que se pretende.

c) Un tercer componente que convierte a la estrategia en flexible y creativa es la secuencia adaptativa. Eso significa que el diseño inicial puede sufrir modificaciones en función de los sujetos, contenidos, condiciones espaciotemporales, agrupamientos, situaciones nuevas que aparecen a lo largo del proceso. Nosotros mismos hemos constatado en diversas ocasiones que las estrategias docentes utilizadas con un turno o grupo de alumnos funciona y con otro no. Una estrategia tienen tanto de sucesión planificada de acciones como de indeterminación sociológica.

d) La realidad contextual es sin duda un elemento clave por cuanto sitúa la teoría y la acción en la realidad concreta, en la pura complejidad de los hechos en los que confluyen decenas de variantes. "La valoración del contexto tal vez sea el componente más sustantivo y esencial de la estrategia frente a otros conceptos mediadores como método, procedimiento, técnica... en los que predomina la secuencia encadenada. El contexto es el referente de partida, de proceso y de llegada" (S. de la Torre. Oc, p.114). Podemos describir perfectamente todos los elementos anatómicos, fisiológicos y psicológicos de una persona; pero cuando ésta actúa, lo hace como un todo que se adapta a las circunstancias.

e) Los agentes o personas implicadas tienen un papel determinante en los logros o frustraciones. Una estrategia no es sólo acción, sino acción lleva a cabo por personas y en función de su grado de implicación, entusiasmo, convicción... los resultados son unos u otros. La actitud de las personas implicadas juega un papel decisivo en la dinámica, clima, grado de satisfacción y resultados. Una misma estrategia desarrollada por un profesor/a u otro tendrá efectos bien distintos en los estudiantes.

f) Por fin, es preciso hablar de la funcionalidad y eficacia; esto es de la pertinencia y eficacia que le otorgue validez para lo que se pretende. De este modo, al final, metas y logros se encuentran ante la conciencia reflexiva de la pertinencia ética y validez o utilidad en condiciones semejantes, porque la estrategia no es buena o mala, en general, sino adecuada o inadecuada para lo que pretendemos. Y esta utilidad, en didáctica no acaba con el logro, sino que tratamos de que pueda ser utilizada por otras personas, situaciones con iguales resultados. Por eso, la estrategia transciende a su vez el caso concreto. No termina con él, sino que se toma como referente para recurrir a ella debido a su validez y utilidad. En otras palabras a su funcionalidad y eficacia.

En resumidas cuentas, la estrategia didáctica comporta toma de conciencia de las bases teóricas que la justifican y legitiman, concreción de la intencionalidad o meta, secuenciación de acciones a realizar de forma adaptativa, determinación de roles o funciones de los agentes implicados, contextualización del proceso y consecución total o parcial de logros. 


\section{Descripción general del modelo holodinámico para analizar las estrategias}

El modelo que proponemos intenta integrar de forma dinámica e interactiva los ocho componentes que conforman el proceso de enseñanza-aprendizaje: seis en situación de aula o dos anteriores y posteriores. Nos han servido de referencia los trabajos de comunicación no verbal de D. Hennings (1989), los modelos de enseñanza de B. Joyce y M. Weil (1985), la comprensión del aprendizaje en el aula de N. Entwistle (1988), la construcción y análisis de procesos de enseñanza de L. M. Villar (1999), el desarrollo profesional del docente de P. de Vicente (2002).

Desde una perspectiva interactiva y psicosocial interesa saber no sólo lo que pasa en el aula, sino por qué pasa, quién lo determina, cómo afecta a los participantes y sobretodo qué cambios provoca en alumnos y profesor. De ahí que la observación se complemente con la entrevista. Este modelo de análisis nos permite además determinar el perfil docente y contribuir a la reflexión y formación docente en sus tareas académicas y profesionales. Es un modelo holodinámico y multidimensional por cuanto además de servir para describir la realidad del aula nos da explicaciones en base a las interrelaciones de los componentes. La presentación formal es reflejo de la interacción de modo que a través del clima se conectan todos los componentes.

Las ocho dimensiones que conforman nuestro modelo observacional y explicativo son:

1. Supuestos implícitos del docente. Lo que piensa, siente, cree, planea el docente

2. Contexto del aula

3. Rol docente

4. Rol discente

5. Organización

6. Recursos

7. Clima

8. Evaluación

Si bien es cierto que la estrategia viene conformada en su totalidad mediante la integración de las diferentes dimensiones, el carácter holodinámico e interactivo nos permite aproximarnos a la misma a través de cualquiera de ellas. Esto es, a través del rol docente o discente, del clima o de la organización, podemos intuir el tipo de estrategia de que hablamos, pues al estar relacionadas, se implican de alguna forma.

Un modelo de análisis como el propuesto además de facilitarnos el análisis de cuanto sucede en el aula desde el punto de vista didáctico, posee indicadores suficientes para llevar a cabo propuestas didácticas innovadoras. No siempre se tendrá información completa de todos los indicadores, pero nos abre la puerta para tomar en consideración algunos de ellos en nuestra práctica docente. Estamos ante un modelo polivalente en sus dimensiones, holístico en sus efectos formativos, recursivo entre análisis y aplicación, reflexión y acción, interactivo por cuanto los elementos descritos no aparecen por separado en el aula, sino concurren de forma simultánea e interactiva. No se puede hablar de rol docente sin referirse al tiempo del estudiante, ni de las condiciones contextuales y recursos al margen de los aspectos organizativos y de ninguna de ellas sin referirnos al clima.

Dame una estrategia adecuada y alcanzaré cuanto deseas. Las estrategias son el punto de apoyo y la palanca para alcanzar objetivos y producir cambio. 
MODELO DE ANALISIS DE ESTRATEGIAS DOCENTES EN AULA

\section{EDIFID}

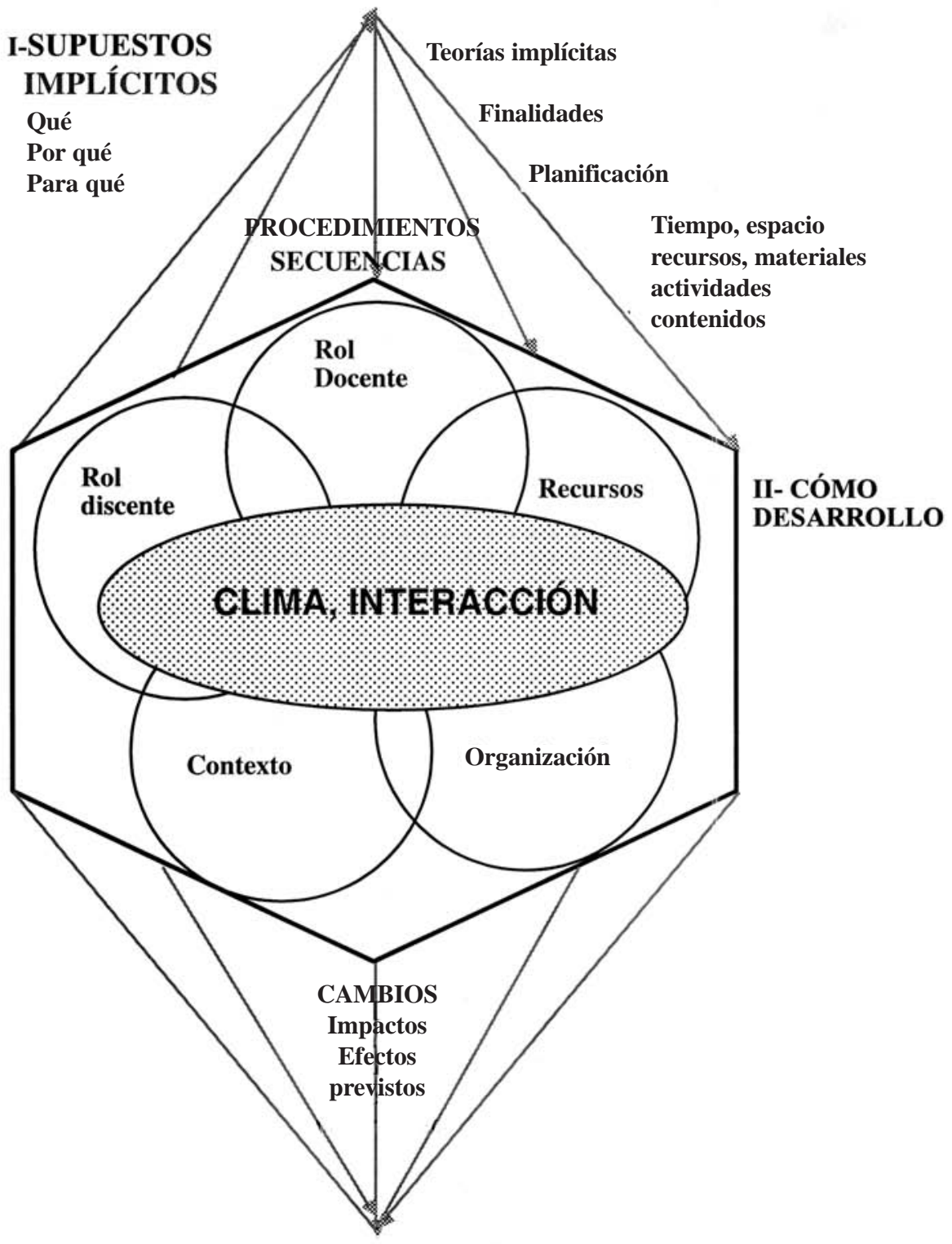

III- EVALUACIÓN 


\section{Metodología de estudio de casos y perfiles de estrategias docentes}

La investigación que presentamos forma parte de un Proyecto mucho más amplio basado en la integración de tres conceptos capitales en educación: investigación, innovación y formación. Estos tres conceptos, que habitualmente se estudian de forma independiente, cohabitan en este proyecto. En este sentido planteamos la generación de conocimiento con el fin de promover cambios de mejora en la enseñanza que a su vez determinan la formación docente y desarrollo profesional. En cierto modo es como un holograma o realidad tridimensional en la que se puede contemplar al tiempo las tres vertientes de modo que cada una de ellas implica y determina a las otras dos de igual modo que en un volumen, la altura, la profundidad y la anchura se requieren con igual importancia. La ausencia de cualquiera de ellas impediría la percepción de un volumen; la presencia conjunta de las tres determina la existencia del concepto volumen. En este sentido, el desarrollo y mejora de la calidad educativa (el cambio) se facilita cuando se contemplan al tiempo la investigación, la innovación y la formación docente.

El error de la pedagogía tradicional es el haber fragmentado tanto el conocimiento que ha desmembrado los saberes de su sentido. El saber que proporciona una investigación no acaba en sí misma, sino en su utilidad o uso, en lo que se haga con esa información, en los cambios que genera. De igual modo la innovación produce cambios sustantivos en tanto mejoran los procesos, los resultados y las personas implicadas en dicho proceso: profesorado, alumnado, agentes externos. Por otra parte, contemplar la formación docente desde modelos autónomos, desconectados de lo que es la investigación y la innovación, no suele producir cambios importantes en los centros educativos. Es decir, el objetivo central de esta triangulación conceptual es generar cambios constructivos.

La metodología de investigación que aplicamos en esta ocasión es el estudio de casos. Para Stake (1999), el estudio de casos nos proporciona una visión más global y detallada a la vez que proveernos de herramientas que nos permite examinar en profundidad los aspectos fundamentales del fenómeno. Para Cohen $(1990,164)$ el investigador del estudio de casos observa las características de una unidad individual, ya sea un individuo, un grupo, una escuela o una comunidad.

El propósito de la observación en el estudio de casos, comenta G. Pérez (1994, 81) consiste en probar de modo profundo y analizar con intensidad el fenómeno que constituye el ciclo vital de la unidad con el fin de obtener ideas relevantes que puedan ser trasladas a otros casos o situaciones. Busca la comprensión del fenómeno de cara a una posible modificación y mejora.

El estudio de casos, comentan Latorre, Rincón, Arnal $(1996,233)$ es la forma más pertinente y natural de las investigaciones idiográficas desde una perspectiva cualitativa y debe considerarse como una estrategia encaminada a la toma de decisiones. Su verdadero potencial radica en su capacidad para generar hipótesis y descubrimientos. Nosotros añadimos en su facilidad para generar cambios en las personas implicadas por cuanto se alcanza un grado de concienciación que no se obtiene a través de otras estrategias. Es una metodología heurística que facilita la solución de problemas y los sujetos se convierten en actores.

El investigador, comentan los autores mencionados, a medida que va descubriendo las fases del estudio incorpora las nuevas ideas y planteamientos que van surgiendo, lo que permite modificar o reestructurar las anteriores. Este proceso es 
recurrente a lo largo de todo el estudio, por cuya razón nosotros lo incluimos en lo que calificamos de metodología de desarrollo.

Una metodología de investigación basada en el desarrollo la entiendo como el proceso de construcción del conocimiento en el que se retoman en diferentes momentos y de forma interactiva y recursiva los objetivos, las estrategias y las valoraciones con el fin de aproximarse al estudio del cambio. Este procedimiento de carácter abierto y recursivo es válido tanto para la construcción individual como colectiva del conocimiento por cuanto uno y otro son fruto de la relación entre los conceptos ya adquiridos y los nuevos conceptos a través de los estímulos del medio (Torre, 2003).

El estudio de casos que utilizamos en la presente investigación es de carácter descriptivo, múltiple, aplicada a nuestra investigación, la unidad de observación, análisis y comprensión es del desarrollo en el aula de una estrategia didáctica. Por tanto intentamos recoger la máxima información en torno a lo que sucede en el aula, pero también al pensamiento de los agentes principales que son el profesorado y el alumnado sin olvidar el contexto, los recursos, el clima, los elementos organizativos, etc. Así pues, nuestro foco de atención es lo que sucede durante una o más sesiones de clase que conforman una micro o macro esctrategia, en los términos que ya quedó definida en páginas anteriores. De ahí extraemos informaciones relevantes de cara a mejorar la práctica docente.

La elaboración de perfiles conectados al estudio de casos es una de las aportaciones significativas de la presente investigación. El sucesivo estudio de casos nos permite aproximarnos a modalidades docentes y a describir lo que denominamos perfiles de estrategias docente en base a los ocho parámetros del modelo. Los perfiles son representaciones que permiten comparar gráficamente diferentes casos o grupos en base a una misma escala. Esta estrategia se ha utilizado frecuentemente en el campo diagnóstico y psicopedagógico. Este tipo de representación permite una interpretación rápida y útil para estudiar los factores de éxito al tiempo que orientar al docente sobre los puntos susceptibles de mejora.

Un perfil más abierto, tendente a altas puntuaciones pondría de manifiesto el buen funcionamiento del proceso enseñanza-aprendizaje. Un perfil en el que alguno de los parámetros resulta menos valorado, es un motivo de análisis posterior. Por otra parte, constatamos que aun apareciendo menos valorados indicadores como recursos, organización o elementos contextuales, el alumnado expresa un alto grado de satisfacción. Eso muestra que el grado de satisfacción o insatisfacción tiene que ver más con la interacción, el clima y el saber hacer docente, que con elementos materiales y formales. Los perfiles nos proporcionan una visión global de lo que entendemos por estrategia en la práctica.

La fuente de información de la que se nutren los perfiles son: entrevista al profesor-a, entrevista o cuestionario al alumnado, observación del aula, informe del observador, grabación de la sesión o sesiones, comentarios realizados en la parrilla de observación, visionado de la grabación y vaciado en una parrilla de análisis construida a tal efecto por el equipo de investigación. Toda esta información se vuelca en el estudio de caso y se representa gráficamente en el perfil. Es por ello que los perfiles no son solamente fruto de un promedio estadístico, como pudiera ocurrir en el ámbito psicopedagógico, sino que sintetizan mucha información cualitativa, contrastada mediante el procedimiento de jueces o evaluadores diferentes. 
Una vez el equipo elabora el informe de caso, se hace llegar copia del mismo al profesorado colaborador quien en ocasiones se ha puesto en contacto con el equipo para discutir los resultados.

\section{El cine formativo como estrategia docente innovadora. Orígenes y actualidad}

Los antecedentes de esta estrategia se sitúan en el Seminario de Cine Formativo, formado por profesorado de las Facultades de Pedagogía y de Formación del Profesorado en 1995, quienes se vienen reuniendo periódicamente para realizar, en los últimos diez años, una tarea de formación en lenguaje cinematográfico y de reflexión sobre los valores educativos del cine, con la finalidad de poder difundirlos a través de una Semana de cine formativo en la que cada año se desarrolla una temática formativa diferente. Se han tratado temas como: Los medios de comunicación y el cine formativo, el cambio a través del cine, aprender del conflicto en el cine, el tratamiento de la diversidad a través del cine, la resiliencia en el medio cinematográfico, las emociones en el cine, la creatividad y el cine, entre otros.

Desde la perspectiva de formar para la vida, el cine representa un fenómeno de gran interés educativo y social. Puede ser considerado como un fenómeno de multitudes, como espectáculo, como cantera de artistas, como negocio que mueve millones, como evasión y ocupación del tiempo libre, pero también como entorno formativo, como estrategia para aprender y estudiar los cambios de personas e instituciones. El cine puede ser un poderoso instrumento para cambiar los valores sociales. El cometido del Seminario es ir aprendiendo del cine en cuestiones como el cambio formativo, la comunicación, la creatividad, el conflicto, la diversidad, la multiculturalidad, el aprendizaje integrador... En suma, el cine hace de puente entre la formación y la vida y este espacio es el que desarrolla el Seminario.

Diez son las Semanas de Cine formativo que se han realizado hasta el 2004. En noviembre de 1995, con motivo de la conmemoración de la primera proyección cinematográfica, el Seminario decidió organizar la primera Semana de cine formativo, celebrándose, a partir de entonces, cada curso, sin interrupción y con incremento de éxito, hasta la actualidad. Con ellas se pretende ir más allá de una simple actividad pedagógica, quiere superar el debate sobre el sentido educativo y didáctico del cine. Con esta semana anual pretendemos sensibilizar al alumnado universitario sobre el poder formativo/deformativo de los medios de comunicación de masas. Algunos objetivos son:

1. Mostrar una vía superadora de la confrontación teoría - práctica, mediante la integración de la observación, reflexión y aplicación. La visualización y debate, la organización de conferencias y mesas redondas proporcionan un modelo formativo innovador.

2. Profundizar en los componentes formativos del cine, aprendiendo a verlo en clave formativa. El procedimiento didáctico Observar - Relacionar - Aplicar (ORA) facilita la lectura formativa de muchas películas comerciales. Esta lectura puede hacerse desde la Pedagogía, la Psicología, la Sociología, la Filosofía, la ética, la Historia, la Lengua y Literatura, el Arte, el Derecho..., por no mencionar otros ámbitos.

3. Tomar conciencia de que el cine, y más concretamente el cine televisivo, está contribuyendo a fomentar, transmitir, afirmar o cambiar valores y contravalores. 
Dado el volumen de películas que se emiten anualmente, podemos considerar que estamos ante un medio con una enorme influencia sociocultural.

La Semana del Cine formativo ha ido adquiriendo una entidad propia en el contexto universitario y ha ido aumentando su aceptación y la participación de estudiantes y profesionales internos y externos a la universidad. Su proyección hacia el futuro viene dibujándose en tres direcciones:

1. Desde la perspectiva curricular. Desde 1999, existe la posibilidad de obtener reconocimiento de créditos $(3 \mathrm{cr}$ ) mediante la participación y realización de algunos trabajos, por parte del alumnado que acude a este evento. La constitución de una asignatura de cine formativo, muestra, también, el interés despertado. En este ámbito, señalar, la importancia que ha adquirido el cine formativo como estrategia didáctica y la investigación que sobre ella se está llevando a cabo, en un contexto amplio, como el del proyecto EDIFID.

2. Desde la proyección a través de publicaciones. Se han publicado libros surgidos del trabajo en Seminario y de los materiales curriculares elaborados para el desarrollo de las sesiones de cine durante las Semanas de cine educativa que se han celebrado. Entre ellos citamos: Torre, S. de la (Coord) Cine formativo. Octaedro; Torre, S. de la (Coord) Cine para la vida. Octaedro; Torre, S. de la (Coord) Aprender del conflicto en el cine. PPU.

3. Desde la difusión y participación en otros proyectos. Se ha solicitado del Seminario de Cine formativo su participación en la organización de Semanas de cine similares en otras instituciones. Así, con temática relativa a la interculturalidad en el cine, el Instituto Iberamericano de Cooperación ha solicitado la colaboración del Seminario para el asesoramiento en la organización de eventos similares.

Desde esta perspectiva, presentamos una de las estrategias investigadas por el grupo GAD del Departamento de Didáctica y Organización Educativa de la Facultad de Pedagogía de la Universidad de Barcelona: el cine como instrumento educativo al servicio de la formación inicial del profesorado en las Facultades de Pedagogía, de Formación del Profesorado, estando abierta a la formación permanente de docentes y profesionales relacionados con el mundo de la educación.

De las estrategias estudiadas por el grupo EDIFID, cabe destacar El forum como estrategia evaluativa, el diálogo analógico, los proyectos integradores, la utilización de películas comerciales, entre otras.

\section{Estudio de caso: El cine comercial como estrategia didáctica'}

Para poder responder a los interrogantes, que en forma de propósitos, el grupo GAD se ha planteado en su trabajo de investigación sobre las estrategias didácticas innovadoras utilizadas por el profesorado de nuestras Facultades, se han observado y

1. Esta es una estrategia concreta a través de la cual, queremos ilustrar tanto la metodología de caso aplicada a las estrategias didácticas, como a la mejora de la acción docente en el aula. Otras estrategiascaso en curso fase de investigación son: el diálogo analógico, proyecto como estrategia integradora de aprendizajes, día de la palabra, el relato como estrategia didáctica, estrategias audiovisuales, estrategia expositiva-ilustrativa, el vídeo como catalizador de aprendizaje inferencial... 
analizado las estrategias más habituales en materias obligatorias y optativas, de mañana y de tarde, para conocer cómo enseñan aquellos profesores bien evaluados por el alumnado, de forma que podamos obtener algunos indicadores de éxito en la enseñanza y en el aprendizaje y extraer el perfil de preferencias del alumnado, para contrastar la satisfacción en los aprendizajes con la metodología docente.

Por otra parte se han descrito las formas de actuar, en forma de estudio de casos y se han elaborado nuevas estrategias metódicas que, una vez implementadas, ofrezcan la posibilidad de investigar sus efectos y los cambios producidos. De este modo tratamos de elaborar un modelo de formación universitaria innovador y comprensivo, difundiendo los resultados con el fin de hacer una aportación a la demanda de mejora de la calidad de la docencia universitaria.

De todos los estudios de caso investigados, hemos elegido el cine como estrategia docente innovadora en un curso de doctorado, realizado en el curso 2002-2003, a que además del interés que despertó en el alumnado, supone una visión microestratégica que complementa el desarrollo macroestratégico llevado a cabo en las Semanas de cine formativo que el grupo GAD viene desarrollando desde 1995. Son dos caras de una moneda que amplían el potencial de esta estrategia docente. Por ello pasamos a describirla, en su modalidad de aula, en el contexto de un seminario de doctorado, con la complicidad de profesor y alumnos que es la mejor relación que se puede establecer en las aulas donde fluye el conocimiento y la motivación.

\section{Ficha Técnica}

\begin{tabular}{|l|l|}
\hline $\begin{array}{l}\text { Profesor: } \\
\text { Saturnino de la Torre }\end{array}$ & $\begin{array}{l}\text { Asignatura: Estrategias Didácticas y Medio Formativo } \\
\text { Tipo: } \text { Optativa }\end{array}$ \\
\hline Carrera: Doctorado & Turno: Tarde \\
\hline Curso: $1^{\text {er }}$ curso & Aula: Seminario Doctorado \\
\hline Duración clase: 180 minutos & Fecha: 17 febrero 2003 \\
\hline № alumnos: 9 & \\
\hline
\end{tabular}

\subsection{Descripción del contexto de la clase}

Describiremos y complementaremos los datos especificados en la ficha técnica, de manera que se pueda entender, comprender y contextualizar la estrategia utilizada y la actuación docente.

La estrategia se basa en el apoyo de medios, en este caso el vídeo, para propiciar una enseñanza donde el "transmisor" de contenidos sea precisamente el medio citado, más las debidas intervenciones del profesor.

El docente, catedrático con larga experiencia en la enseñanza universitaria, muestra un dominio tanto del contenido como de la metodología didáctica. Experto y profundamente dedicado a la creatividad, con un amplio dominio de la temática objeto de la sesión, motiva con su propio entusiasmo al resto del alumnado para que participen y contribuyan al ámbito con sus aportaciones.

La asignatura pertenece al programa de doctorado del Departamento de Didáctica y Organización Educativa. Al ser una asignatura de un itinerario concreto, 
el alumnado la escoge según sus intereses, por lo tanto partimos desde un inicio con un alto grado de motivación. Además, cabe recordar que las clases de doctorado suelen ser poco numerosas, lo que favorece a una mayor interacción. En cuanto al contenido de la asignatura "creatividad y medio formativo", podríamos decir que tiene diferentes centros de interés, lo que requiere un alto nivel de conocimiento y estructuración por parte de profesor.

Semestre, se imparte durante el segundo trimestre del primer curso, lo que implica entre otras cosas, que el alumnado ya ha adquirido cierto ritmo de trabajo y se mueve más según los intereses de su posible proyecto de tesis.

El alumnado procede de carreras diferentes - educación física, pedagogía... - y también hay cierta heterogeneidad en los niveles - algunos/as han realizado masters, postgrados... - . Hay que considerar que la mayoría comparten estudios con trabajo, esto conlleva en muchas ocasiones falta de tiempo para realizar las tareas académicas. Se evidencia, en ocasiones, la presión que siente el alumnado por la realización del proyecto de tesis.

La clase, de tres horas de duración, se inicia a las 18:30 y finaliza a las 21:30. Suele darse un descanso de 10 a 20 minutos alrededor de la mitad de la sesión.

\section{Descripción de la estrategia en el aula. Su desarrollo}

El profesor realiza una exposición inicial donde expone el objetivo de la sesión. A continuación, se procede al visionado del vídeo, donde el profesor pasa a ser un espectador más. Al finalizar la proyección, adquiere un rol más estimulador y orientador para obtener un alto grado de participación en el debate final. Estamos, por tanto, ante un ejemplo de estrategia de índole participativa con apoyo en el medio audiovisual.

Para ver como se concreta esta estrategia en la práctica, nos referiremos a los siguientes puntos en la descripción del perfil:

1) Supuestos implícitos

2) Contexto del aula

3) Recursos utilizados

4) Rol docente

5) Rol discente

6) Aspectos organizativos

7) Clima del aula

8) Valoración de la sesión

\section{1) Supuestos implícitos}

De la entrevista con el profesor ST se infiere una clara intencionalidad de lo que pretende y cómo lo pretende, así como de su concepción pedagógica en torno al cine formativo, al aprendizaje integrado y al sentipensar. Sentipensar es un concepto que está presente en su lenguaje de forma habitual y que lo entiende como "Proceso mediante el cual ponemos a trabajar conjuntamente el pensamiento y el sentimiento. Es la fusión de dos formas de interpretar la realidad, a partir de la reflexión y el impacto emocional, hasta converger en un mismo acto de conocimiento que es la acción de sentir y pensar". 
¿Qué pretendía en esta sesión?

"En la sesión pretendía ilustrar una estrategia didáctica basada en la utilización del cine como recursos desencadenante de reflexión, participación, implicación en los procesos de aprendizaje. Demostrar que la formación no está tanto en la información sistematizada para la instrucción sino en la intencionalidad del docente y discente para compartir significados. Mediante el relato cinematográfico, en nuestro caso la película El Cartero y Pablo Neruda pretendía que el alumnado constatara cuatro tipos de procesos formativos:

a) El proceso de aprendizaje personal, tanto en conocimiento como en actitudes, valores y emociones. Expresar lo que cada uno siente y piensa a raíz del visionado de la película. Ofrecer un ejemplo de "sentipensar".

b) Proceso de construcción de conocimiento, de concepciones o teorías como la del cambio, la creatividad, la enseñanza, etc. Cómo se construye el conocimiento científico mediante la formulación de supuestos y constatación de problemáticas.

c) Proceso de innovación, utilizando el cine como una estrategia innovadora.

d) Proceso de creación o generación de textos, composiciones o realizaciones a partir de lo sugerido en la película. Se ilustra esta parte mediante algunas poesías.

¿Y qué añade la utilización del cine a una clase basada en información verbal o en documentales? Usted inició de forma sistemática el cine formativo mediante el modelo ORA (Observar, Relacionar, Aplicar), pero en qué está su novedad o innovación.

Mediante el modelo ORA podemos valernos del cine para aprender cuatro tipos de procedimientos, al tiempo que adquirir un aprendizaje integrado. Cómo ver una película para aprender a aprender y aprender a enseñar, aportar algo nuevo al conocimiento existente, Ilevar a cabo innovaciones, aplicar la creatividad. Son cuatro procesos que se completarían con lo que sería el aprendizaje integrado, en el que convergen componentes cognitivos, de habilidad, psicosociales, conativos al tiempo que nos proporciona un aprendizaje referido a la vida. La riqueza cultural generalmente fragmentada en asignaturas, núcleos, temas, adquiere in significado relevante (integra lo académico y lo cotidiano) e intuitivo, al verse conectadas a través del relato la historia, la geografía, la filosofía, la psicología, el comportamiento humano, la sociología, la política, la economía... y cuantas disciplinas se vienen trabajando aisladamente.

El cine viene a ser como el cuerpo humano. Aunque se estructura en sistemas, órganos, funciones, miembros... todo ello tiene sentido y unidad cuando actuamos, hablamos, pensamos o sentimos. El sistema muscular, óseo, sanguíneo, nervioso, pensamiento... cobran sentido cuando escribo. Pues bien, en una película se dan cita muchos de esos saberes que si bien mantienen su propia identidad epistemológica, están interrelacionados desde un punto de vista sociocultural, de crecimiento personal, y desarrollo profesional. 
¿Tiene que ver el cine con lo que denomina sentipensar?

Bien, el cine tiene un efecto de integración, por lo tanto los mensajes llegan a través de diferentes lenguajes como la palabra, la música, el movimiento, el relato, y por supuesto la imagen. Eso quiere decir que el cerebro utiliza muchas más zonas, activa muchas más neuronas para captar el significado que proviene por cada uno de los lenguajes, significa que pone en juego no solo procesos lógico-deductivos vinculados al hemisferio izquierdo, sino que también activa el hemisferio derecho a través de la música, el espacio, la imaginación, las emociones... Es por ello que el relato fílmico tiene un efecto de desarrollo cerebral, congitivo-emotivo, muy superior al de cualquier otro sistema de información. El cine estimula todo el cerebro y una buena película que hace pensar, que comporta valores, que crea dilemas, tiene un potencial formativo muy superior a cualquier lección. Por lo que trasmite, por lo que sugiere y por lo que hace pensar y sentir. De ahí que lo considere como un excelente recurso para "sentipensar".

¿Y cómo se concreta eso en la película El cartero y Pablo Neruda? ¿Por qué eligió esa película para mostrar los cuatro puntos de que habló: aprender, construir conocimiento, innovar y crear?

Esta película es una fascinación desde muchos puntos de vista. Tal ves sea una de las pocas que considero extraordinariamente formativa. En el relato, en el poder de la metáfora y la palabra para transformar a las personas, en la actuación de los personajes principales de Neruda y el Cartero, en la sensibilidad, en las emociones, en el humor, en la música, en la personificación del mar que alcanza categoría de personaje. Bueno, no terminaría. Desde un punto de vista educativo se puede analizar el proceso de cambio del cartero en siete etapas o momentos descritos en la película, se puede abordar el tema de la creatividad como persona, proceso, ambiente y resultado, se puede analizar la relación transformadora entre formador (Neruda) y formando (el cartero). Se pueden inferir muchos conceptos como los indicados, pero también elaborar modelos y teorías sobre la creatividad. En ese sentido nos permite no solo aprender, sino construir conocimientos y por supuesto crear. La película invita a crear diálogos sobre la palabra, el silencio, el amor. Para no extenderme me remito al artículo tal vez más hermoso que yo haya escrito en mida. El título es "El Cartero y Pablo Neruda o el poder de la palabra creativa".

¿Cómo se desarrolló la clase según su opinión?

La clase se desarrolló según lo previsto, con falta de tiempo, pero con una implicación y participación más que aceptable. Hubo aportaciones orientadas hacia la vivencia y lo emocional, hacia el impacto de la película y otras más centradas en las ideas y procedimientos. Se generó un excelente clima comunicativo y las potencialidades educativas y creativas de la película.

De hecho la sesión de 3 horas, con proyección incluida, se centra en la estrategia de utilización de recursos audiovisuales como inductores de aprendizajes. La sistematización de la estrategia lo comento en otro momento. 
¿Como valora el clima de participación e implicación del alumnado? ¿Cree que se consiguió ese clima que pretendía?

El clima, que para mi es la pieza clave de cualquier estrategia didáctica innovadora y creativa, fue altamente constructivo. Hubo participación espontanea y sentida de todos los participantes. Hubo expresiones de emoción y sentimiento respecto al significado y alcance de la película. Realmente, desde mi particular punto de vista, o esa es la impresión que tengo, fue una clase en la que la construcción de los aprendizajes se basó en la interacción entre los asistentes, de modo que el profesor fue un mero inductor y coordinador de intervenciones. Un ejemplo de construcción colaborativa de aprendizajes y conocimientos compartidos.

\section{2) El contexto del aula}

El aula dispone de las condiciones suficientes para desarrollar una estrategia basada en recursos audiovisuales. Dispone de retroproyector y vídeo fijos. En cualquier momento de la clase puede hacerse uso de los mismos. Las condiciones ambientales son propicias y el espacio amplio. Permite mucha movilidad y agrupaciones diferentes, ya que tanto mesas como sillas no son fijas. El aula está adecuadamente equipada y es flexible en su mobiliario. Faltan, eso sí, recursos informáticos permanentes en la clase.

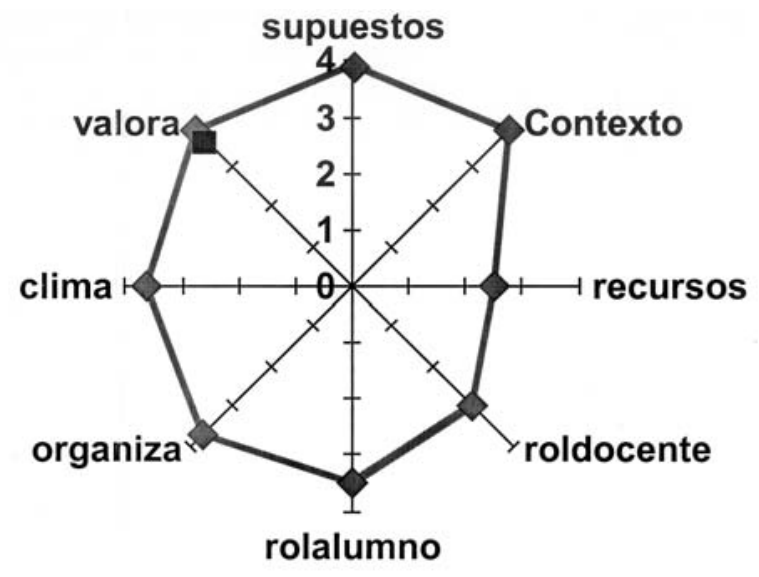

Perfil de estrategia docente, con apoyo en recursos audiovisuales

\section{3) Recursos utilizados}

La variedad de los recursos utilizados, tanto materiales como analógicos se considera positivo. En el caso estudiado se utilizan recursos audiovisuales - vídeo -, recursos de tipo textual - fotocopias, artículos -, y recursos imaginativos - relatos, poemas -. Conviene remarcar que cuando hablamos de recursos nos limitamos muchas veces a los instrumentos materiales o tecnológicos olvidando que los analógicos, imaginativos, lúdicos, suelen tener un efecto importante en la motivación y desarrollo de la creatividad. Recursos como la música, el relato, el caso, el lenguaje poético, facilitan los procesos de "sentipensar" y por tanto de conectar pensamiento y sentimiento. 
No hace uso de la pizarra - tal vez uno de los recursos más utilizado por el profesorado en general - ni de transparencias. Y consigue que, en este caso el vídeo, no se convierta en el sustituto del profesor como "transmisor" del conocimiento, si no que realmente se trata de una estrategia con apoyo en un medio audiovisual tan integrador como es el cine. Por si sola la película tiene un poder sugestivo, implicativo y de aprendizaje no sólo cognitivo sino emocional. Al proyectar en clase, tiene un efecto diferente de verla cada uno en casa o verla de forma colectiva. Pasa a ser audiovisual paradigmático y ejemplificador. Los comentarios del profesor contribuyen a verla desde una óptica formativa. En este caso se utiliza un recurso, pero representa una integración de recursos.

\section{4) Rol docente}

Si vamos analizando punto por punto las diferentes categorías observamos que en referencia a la presentación, destaca muy positivamente la motivación inicial así como también el objetivo y presentación de la sesión.

Resalta la interacción comunicativa. No se trata en absoluto de una clase magistral donde predomina una comunicación verbal unidireccional, si no que al contrario, la mayor parte del tiempo se está dando una comunicación bidireccional en valores emotivos y reflexivos, es más, podríamos incluso llegar a afirmar que la comunicación no es tan sólo bidireccional si no que ampliaríamos el término a multidimensional por cuanto el relato fílmico está como referente en el debate que sigue a la película y los comentarios se cruzan entre todos los asistentes.

En cuanto a la comunicación no verbal, pese a que la expresividad en el rostro y la gesticulación no están muy presentes en el profesor, ni tampoco la movilidad por cuanto al ser pocos estamos todos sentados, si que utiliza frecuentemente otros recursos como son los silencios, las variaciones de tono, el paralenguaje, los gestos...

Predomina sin lugar a dudas un estilo docente implicativo, estimulador y creativo.

Referente a la metodología los adjetivos que la describen son: motivante, estimulante, ordenada, clara, buena secuenciación de ideas, intuitiva, visual, con ejemplos, participativa, implicativa, grupal, basada en medios... En definitiva, una metodología que se aleja absolutamente de la tradicional figura del profesor rígido, sin flexibilidad, y que se basaba en un metodología expositiva. Está presente cuando interviene la vivencia de lo que dice, la dimensión emocional. Siente lo que expresa y esto suele llegar al que escucha.

Los contenidos, acorde con la metodología tan "dinámica" descrita anteriormente, son de actualidad, despiertan interés y se hacen - con la ayuda del profesor comprensibles, y fáciles de entender.

\section{5) Rol discente}

La sesión se divide en tres momentos bien diferenciados: la exposición inicial por parte del profesor que explica el sentido de la sesión, el visionado de la película "El cartero y Pablo Neruda", y el debate final.

En la primera parte, el rol del alumnado es pasivo, receptivo de la información del profesor, puesto que toda la importancia y la carga formativa de la sesión están en la exposición del profesor. 
Durante la proyección de la película predomina el silencio, salvo algún comentario del profesor referido a una escena que para él es clave: el encuentro en la playa donde Neruda le explica el sentido de la metáfora y él se siente capaz de utilizar dicho ansiado lenguaje, cautivador de las mujeres. Sin embargo, a pesar del silencio verbal, no hay pasividad. Hay un clima de implicación afectivo - cognitiva con el relato de la película, una identificación con los personajes que es clave del cambio interior. Los alumnos ríen con frecuencia, lo cual comporta vivenciar lo que están viendo. Digamos que no hay intervenciones pero se da un grado de implicación superior al que acontece cuando se está escuchando al profesor en una clase magistral.

En el debate que tuvo lugar al terminar la proyección es donde el rol del alumnado llega a su máxima consideración, superando en cierto modo al rol del profesor. En esta parte de la sesión el alumnado es especialmente activo y participativo, la interacción del grupo - clase es óptima, favoreciendo de esta manera al objetivo de la sesión. Los asistentes expresan lo que han sentido, lo que han pensado, lo que les sugiere y la fuerza e interés que han visto en la película.

\section{6) Aspectos organizativos}

Como queda reflejado en la gráfica del perfil, el aspecto de la organización es uno de los puntos más fuertes de la sesión. Este aspecto hace referencia al tiempo, el espacio, los recursos, y las actividades de aprendizaje.

Hay una buena planificación del tiempo, a pesar de que la exposición inicial se alarga un poco más de lo previsto. La distribución general a lo largo de la sesión, de la exposición, el visionado, y el debate, es la adecuada para realizar una síntesis final. Falta tiempo, sin embargo, al final para un cierre tal como tenía previsto. Pretendía acabar con un poema de Neruda.

Hay un buen aprovechamiento del espacio, y adaptación de este a las necesidades específicas de la sesión.

Los recursos, aunque escasos, son los suficientes para lograr el objetivo propuesto. Una mayor utilización de recursos, en este caso, podría suponer la anulación de la funcionalidad de estos.

\section{7) El clima del aula}

El clima alcanza un nivel muy alto, como se puede observar en el gráfico. Se aprecia que el alumnado se siente cómodo y está interesado en la sesión. Es un grupo con un alto grado de cohesión interna, seguramente debido a que comparten otras asignaturas y están motivados, en cierto modo, por intereses didácticos semejantes. Se aprecia seguridad psicológica como para poder expresar sentimientos.

\section{8) Valoración de la sesión}

Teniendo en cuenta las entrevistas realizadas al alumnado, podemos concluir diciendo que en general la valoración global de la sesión es muy alta. Veamos algunos ejemplos.

En referencia a la valoración de la sesión expresan lo siguiente: "Siento la estrategia como un nuevo valor para estimular el aprendizaje a través de los sentidos, 
especialmente a través de la percepción de lo que ocurre en todo el entorno. Los elementos importantes y más destacados no pasan como aleatorios o sin significado. Al contrario, a través del cine los identificamos y los vivimos con más intensidad".

Con respecto a la implicación en la sesión: "Buena, pues estuve implicada y atenta a la sesión, además exponiendo mi visión y opinión al final de la sesión".

En lo que se refiere a las cosas más significativas aprendidas en la sesión: "Sobre aprendizaje integrado y tipos de procedimientos para valernos del cine mediante el modelo ORA. La dinámica propició percibir la manera como el otro hace su propio pensar".

\section{A modo de conclusión}

El modelo multidimensional de análisis de estrategias didácticas en la enseñanza universitaria es el eje de una investigación sobre la docencia universitaria que, situada en el paradigma de interacción psicosocial o ecosistémico. Pretende incidir en una docencia universitaria de calidad. Lo demanda la sociedad y los profesionales de la educación. Tratamos de ir y venir en una vaivén creativo e innovador que nos vaya retroalimentando, como propone una visión ecosistémica del cambio y generación del conocimiento.

Investigamos las estrategias docentes con una metodología de estudio de casos inscrita en un concepto más amplio de que denominamos "metodología de desarro"lo" entendida como proceso de construcción del conocimiento en el que se retoman en diferentes momentos y de forma interactiva y recursiva los objetivos, las estrategias y las valoraciones con el fin de aproximarse al estudio del cambio. La información se traduce a perfiles pudiendo de este modo representar gráficamente los principales parámetros de una estrategia didáctica, mostrándose de inmediato sus puntos fuertes y débiles. En tal sentido, la investigación brinda una triple aportación: la decisión por integrar los conceptos investigación-innovación-formación, la metodología de desarrollo y la incorporación de la estrategia de perfiles al estudio de caso.

Por lo que respecta a los resultados del presente estudio de caso, constatamos que:

a) Los perfiles nos proporcionan una visión global, intuitiva y útil de lo que entendemos por estrategia didáctica en la práctica.

b) Aun apareciendo menos valorados indicadores como recursos, organización o elementos contextuales, el alumnado expresa un alto grado de satisfacción. Eso muestra que el grado de satisfacción o insatisfacción tiene que ver más con la interacción, el clima y el saber hacer docente, que con elementos materiales y formales.

c) El estudio de casos acompañado con la técnica de perfiles son herramientas útiles en la evaluación de la docencia universitaria.

d) El estudio de casos acompañado de la técnica de perfiles aplicados a las estrategias nos ayudan a comprender mejor la razones del éxito de algunos docentes y en base a ello, mejorar las estrategias de aquellos que se encuentran menos valorados. Esta investigación es una herramienta útil tanto en la formación del profesorado novel como de aquellos que no alcanza niveles satisfactorios. 
Confiamos que las aportaciones de este trabajo sean testimonio en si mismo de que la investigación ha de ir unida a la formación y a la innovación (Modelo IFI) si queremos mejorar la calidad de la enseñanza en cualquiera de sus niveles. Cada profesor puede aplicarse a si mismo este modelo IFI y llevando a cabo una autoevaluación de sus estrategias docentes. Es lo que propugnamos.

Formarse es reflexionar sobre la propia práctica para mejorarla.

\section{BIBLIOGRAFÍA}

BARNETT, R. (1995). Improving higher education. Total quality care. Buckignham: Open University.

BIREAUD, A. (1990). Les méthodes pédagogiques dans l'enseignament supèrieur. Paris: Organisation.

CARAMÉS, J.L. (2000). La nueva cultura de la universidad del s. XXI. Oviedo: Trave.

COHEN, L y MANION, L. (1990). Métodos de investigación educativa. Madrid: La Muralla.

Comisión de Docencia (1995). Didáctica universitaria. Sevilla: Publicaciones Universidad Sevilla.

DAVIDSON, J. (2000). La gestión del tiempo. Madrid: Prentice Hall.

DE VICENTE, P. (Coord) (2002). Desarrollo profesional del docente. Bilbao: IceDeusto.

DEL RINCÓN, D.; ARNAL, J. y LATORRE, A. (1995). Técnicas de investigación en Ciencias Sociales. Madrid: Dykinson.

ELINS, N. (1989). Tiempo y espacio. Revista Educación, 298.

ENTWISTLE, N. (1988). La comprensión del aprendizaje en el aula. Barcelona: Paidós.

FERRER, V. (1994). La metodologia didáctica a l'ensenyament universitari. Barcelona: Universidad de Barcelona.

GARCIA-VALCÁRCEL, A. (Coord.) (2001). Didáctica Universitaria. Madrid: La Muralla.

GIBAJA, R.R. (1993). El tiempo instructivo. Buenos Aires: Aique.

GONZÁLEZ, A.P. (Coord) (2002). Enseñanza, profesores y universidad. ICE-URV: Tarragona.

HENNINGS, D. (1987). El dominio de la comunicación educativa. Madrid: Anaya.

JOYCE, B y WEIL, M. (1985). Modelos de enseñanza. Madrid: Anaya.

LATORRE, A.; DEL RINCÓN, D. y ARNAL, J. (1996). Bases metodológicas de la investigación educativa. Barcelona: Hurtado.

MARCELO, C. (1995) Desarrollo profesional e iniciación a la enseñanza. Barcelona: PPU.

OLIVER, C. (2003). Estrategias didácticas y organizativas ante la diversidad. Barcelona. Octaedro.

PÉREZ SERRANO, G. (1994). Investigación cualitativa. Madrid: La Muralla.

MEC (1993). Monográfico sobre Tiempo y espacio. Revista de Educación, 298. 
ROMERO, C. (2000). El conocimiento del tiempo educativo. Barcelona: Alertes. SNEDDON, I. y KREMER, J. (Eds) (1994). An interprising currículo. Belfast: Hmso.

STAKE, R. (1999). Investigación con estudio de casos. Madrid: Morata.

TEDESCO, J.C. (1997). Uso del tiempo en la sala de clases. Información e innovación en educación. OIE.UNESCO, 92.

TEJADA, J. (2000). El docente innovador. En TORRE, S. y BARRIOS. O. Estrategias didácticas innovadoras. (2ª ed., 2002), 47-61. Barcelona: Octaedro.

TEJADA, J. (2000b). Perfil docente y modelos de formación. En TORRE, S. y BARRIOS. O. Estrategias didácticas innovadoras. (2ª ed., 2002), 16-46. Barcelona: Octaedro.

TEJADA, J. (2002). El docente universitario ante los nuevos escenarios: implicaciones para la innovación docente. Acción Pedagógica, 11 (2), 30-42.

TORRE, S. DE LA (2003). Diseño de la investigación y análisis de resultados. Documento Mimeo. Barcelona.

TORRE, S. DE LA (Coord) (1996). Cine formativo. Barcelona: Octaedro.

TORRE, S. DE LA (1997). Innovación educativa. Madrid: Dykinson.

TORRE, S. DE LA (Coord) (1998). Cine para la vida. Barcelona: Octaedro.

TORRE, S. DE LA (Coord) (2001). Aprender del conflicto en el cine. Barcelona: PPU.

TORRE, S. DE LA y BARRIOS, O. (2000). Estrategias didácticas innovadoras. Barcelona: Octaedro.

TORRE, S. DE LA (2003). Dialogando con la creatividad. Barcelona: Octaedro.

UNESCO (2000). L'educació superior en el siegle XXI. Barcelona: UNESCO.

VILLAR, L.M. (1999). Construcción y análisis de procesos de enseñanza: Teoría e investigación. Barcelona: Oikos Tau. 